\title{
HOTELS ADAPTATIVE E-MARKETING MODEL USING LEARNING STYLES AND VIRTUAL REALITY
}

\author{
Luis Alfaro ${ }^{1}$, Claudia Rivera ${ }^{1}$, Jorge Luna-Urquizo ${ }^{1}$, Elisa Castañeda ${ }^{1}$, Jesús Zúñiga-Cueva ${ }^{1}$ \\ and Francisco Fialho ${ }^{2}$ \\ ${ }^{1}$ Universidad Nacional de San Agustín, Arequipa - Perú \\ ${ }^{2}$ Universidade Federal de Santa Catarina, Brazil
}

\begin{abstract}
Experiential marketing considers various types of experiences, seeking greater consumer satisfaction and therefore greater effectiveness in the marketing action, which associated with intelligent systems and immersive technologies, allow the development of applications for exploring services offered by hotel organizations, with a high degree of customization and adaptation, providing experiences with virtual objects according to the profiles, characteristics and identification of the learning styles of the users, obtained by a diffuse neural network, which processes online the information of the interactions of the users with the system, whose results were correlated with those obtained from the application of the survey for classification of learning styles. The information obtained is processed by a Case Based Reasoning module, for the adaptation to users of virtual experiences and objects related to the marketing strategies of hotel facilities and services.
\end{abstract}

\section{KEYWORDS}

Experiential Marketing, Adaptative E-Marketing, Learning Styles, Immersive Technologies, Neurofuzzy Networks

\section{INTRODUCTION}

Experiential marketing includes customer experiences such as sensations, thoughts, actions, and feelings, and which focuses on more satisfactory experiences and therefore greater effectiveness in marketing-action, focusing on enriching experiences based on the communication of marketing actions through the consumer's senses, in order to influence their decision and relationship with a product, service or brand.

These marketing approaches, associated with Virtual Reality (VR) technology, allow the conception and design of applications that for example, could show landscapes and diverse hotel services, using metaphors (Carbonell, Sánchez-Esguevillas, \& Carro, 2016), through simulation, interaction and reification, making images and other sensory stimuli connected to sensors and actuators, seeking the approach to experiences that are only possible to be lived in the real world, thus notably enriching the sensory experiences.

Nowadays, several Artificial Intelligence (AI) techniques are used by researchers for the design of adaptive and flexible web-based systems for the promotion and sales of products and the provision of services (Soni, Sharma, Singh, \& Kapoor, 2020), such as educational and hotel services, which, associated with the approaches and techniques of Experiential Marketing and immersive VR, can provide users with direct experiences, incorporating explicit and tacit knowledge corresponding to the service offered.

The proposal of the software architecture model of a theoretical adaptive e-Marketing system, which considers the profiles of the potential clients as well as their LS, is identified through a neurofuzzy network. This information is then treated by a Case Based Reasoning (CBR) module for the adaptation of the information provided to the needs and requirements, contributing to an effective interaction of the user with the system, which can influence and support the purchasing decision with a direct impact on the results in the hotel and tourism sector, including social distancing measures derived from the COVID-19 pandemic. 


\section{LITERATURE REVIEW}

\subsection{Marketing and the Experiences}

Marketing is based on the consumer's perception of the product or service they are buying. The concept of experiential marketing considers consumers not only as buyers who want to satisfy their needs and benefit, but also as rational and sensitive human beings who want to try different things and experience. Experience is the central element of experiential marketing. It aims at creating different stimuli such as special areas, atmosphere and new scenarios. Thus, consumers are expected to respond to these stimuli, show active purchasing behaviour, live experiences and have sensations such as pleasure (Alagöz \& Ekici, 2014).

According to Gallo, Townsend, and Alegre (2019), the lived experience also generates a personal increase in the value of goods and this improves the effects of word-of-mouth marketing. In this regard, Winn (1993), proposed the need for further research to use the emerging technologies of VR, which make it possible to build knowledge from direct experiences and not from descriptions of these made by third parties (L. Alfaro, Rivera, Luna-Urquizo, Alfaro, \& Fialho, 2019). From this perspective, intangible services may be easier to promote and sell, as the client may be more sensitive to the impact they could have if there is greater sensory stimulation through the VR technologies used in immersive marketing (Hwangbo, Kim, \& Cha, 2017).

Numerous studies have been published on consumer sensations and behaviour, focusing on the senses of sight, taste, touch, smell and hearing, with sensory perceptions and sensory marketing being an emerging area of research (Krishna, 2012). The sensory experience produced by a marketing action begins to be related to the immersive experience using the different senses of the consumer, in which sensory marketing focuses on generating memories from the sensory experiences. Although it would be ideal for the consumer to be exposed to an experience through all the senses, in practical terms this is not feasible, due to the circumstances in which the service is offered, and the cost and technology related to the service offered.

For intangible products or services, there are several marketing management models, one in particular being the service gap model proposed by Parasuraman, Zeithaml, and Berry (2018), which establishes the gap between the consumer's perception, comparing their expectation with their actual experience, allowing a differential to be established that impacts on their memory and sensations. According to Parasuraman et al. (2018), an expectation is formed from previous experience with the service, explicit and implicit communication and word of mouth. The role that the mind unconsciously acquires in decision making is now considered increasingly relevant, allowing it to be established that purchasing decisions have an emotional component. Based on the above, the following hypotheses are proposed:

H1: A Sensory Experiential Marketing System model applied to the Hotel Sector based on Immersive Technologies, will contribute to the attraction of potential customers in the hotel services sector.

\subsection{Emerging Technologies and Artificial Intelligence (AI) in Marketing}

The impact of digital technologies on the customer's experience involves considering the main technological inventions. For Hoyer, Kroschke, Schmitt, Kraume, and Shankar (2020), the global Web has enabled new communications, interactions, and transactions through an entirely new medium and platform. Mobile platforms have information embedded in a device that can be used for multiple business applications.

Official destination websites are recognized as a channel for providing an authoritative source of information to tourists. These websites have led to a number of investigations into communication, destination matching and sales propositions in virtual environments. These virtual activities generate an enormous amount of strategic data with valuable significance and, offer the possibility of profiling tourists through their tours, which supports the provision of more personalized destination marketing.

AI-based technologies can support clients in understanding and better predicting their own preferences. Virtual assistants, chatbots and robots can help clients select from options, advise them and personalize information. For Kumar, Ramachandran, and Kumar (2020), new technologies such as AI enable a company to deliver the right content to the right customer at the right time.

The following hypotheses are proposed on the basis of what is discussed in this section:

H2. Experiential and sensory marketing can be enhanced using artificial intelligence associated with immersive technologies, allowing the delivery of the right content to the right customer at the right time. 


\subsection{Adaptative Systems in Marketing}

For the modelling of the adaptive e-Marketing system, there are several sources of experience, such as those coming from the Intelligent Learning Environments, like the Intelligent Tutorial System (Alfaro et al., 2020), Pedagogical Agents (Lewis \& Lester, 2018), and the Adaptive e-Learning Systems (Al-Chalabi \& Hussein, 2020), whose characteristics and attributes related to the functionalities and parameters of adaptation, can contribute to achieve experiences and the presentation of virtual objects of the e-Marketing system, according to the profiles, characteristics and expectations of hotel clients or guests. According to Shute and Towle (2003), direct or first person experiences are generated for the user, for contact with the facilities and services provided by a hotel through processes of information exploration, and through the use of VR technologies.

In this perspective Grubišić, Stankov, and Žitko (2015) point out that the most important characteristic of these systems is the capacity to adapt the process of interaction and virtual objects, and they must be flexible to the specific requirements of the users. The characteristics of the client or user, which must be considered in order to develop an adaptive e-Marketing system, is a key decision and a success factor.

Botsios and Georgiou (2008) suggest that cognitive skills, together with LS, are important for an efficient learning or exploration process. This approach was used in several works, such as the one proposed by Triantafillou, Pomportsis, and Georgiadou (2002), which recommends CS-focused adaptations, to which virtual experiences and objects, which allow contact with hotel facilities or services, should be adapted. The adaptation to the client's knowledge consists of focusing on the adaptation of the materials to be explored and the presentation of contents, according to the knowledge the client has about the subject (Esichaikul, Lamnoi, $\&$ Bechter, 2011), in relation to facilities, services, experiences, landscape, environment, etc. The adaptive capacity in e-Marketing systems is one of the important factors in improving their quality and efficiency.

In this work, we focus on the use of a CBR to adapt virtual experiences and objects to the LS that must have been previously identified by a neuro-fuzzy network and to the user's knowledge levels, which come from previous experiences. The following hypotheses are proposed on the basis of what has been discussed:

H3: An adaptive model will optimize the productivity and quality of e-Marketing systems by identifying the LSs and considering parameters of the customer profile for the generation of experiences and presentation of virtual objects through a CBR system.

\subsection{VR, AR and MR Technologies in Marketing}

A commonly accepted definition of VR is: "the use of a computer-generated 3D environment with which the user can navigate and interact, resulting in the real-time simulation of one or more of the user's five senses" (Burdea \& Coiffet, 2003). Gutiérrez, Vexo, and Thalmann (2008), state that the three key elements of VR are sensory immersion, interactive simulation, and implicit interaction. Beck, Rainoldi, and Egger (2019) argue that the type of technology solution for tourism applications can be fully immersive, semi-immersive, and non-immersive, stating that the more immersive the solution, the more complex the technology implementation of the devices and the content. They also argue that the Head Mounted Display, for cost-benefit and immersive capability reasons, is a good solution for VR focused tourism marketing activities.

Based on the aforementioned, the following hypotheses are proposed:

H4: The immersive technologies can contribute to the effectiveness of marketing activities and strategies for the hotel sector.

\section{SOFTWARE ARCHITECTURAL PROPOSAL}

This architectural proposal seeks to generate experiences as a central element of experiential marketing, which aims to create different stimuli such as special areas, atmosphere and new scenarios. Consumers are expected to respond to these stimuli, show active purchasing behavior, live experiences and have sensations such as pleasure and entertainment (Alagöz \& Ekici, 2014). Verhoef et al. (2009) defines the experience as a multidimensional construction, and states that the customer experience is holistic in nature, and includes cognitive, affective, emotional, social and physical responses (Verhoef et al., 2009). The architecture is composed of four modules, as shown in Figure 01. 


\subsection{Immersive Marketing Strategy}

In this module, the defined marketing strategy will be implemented. This includes the formal definition of deadlines, contents, and metrics for the evaluation of the expected results. The strategy requires a virtual content generation module, according to the determination of the types of experiences, objects and virtual content to be created. For example, if the strategy seeks in particular to promote a beach hotel for the summer season, this strategy defines what virtual experiences and objects should be created, and what types of interactions are intended to be used, according to the demands, profiles, and LS of customers.

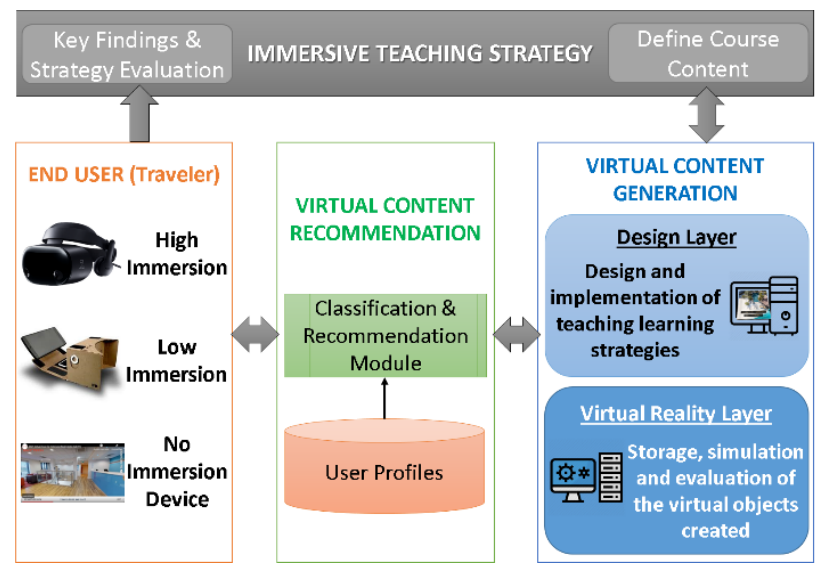

Figure 1. Software Architecture proposal. Source: Adapted from Alfaro, Rivera, Luna-Urquizo, Zuńiga, et al. (2019)

\subsection{Virtual Content Generation}

It provides the structure and links the tools, which allow the generation of virtual objects. This includes the generation of videos and 360-degree simulations, which can be complemented with virtual objects and expand the information and interaction that will be represented in the marketing strategy. This module is responsible for the storage, simulation, evaluation, and testing of virtual content. Evaluation refers to the fact of verifying the fulfilment of the marketing strategy. The simulation corresponds to the behaviour of the solution with respect to different profiles and LS of customers or users.

\subsection{Virtual Content Recommendation}

It represents the logic of the adaptive system, which effectively executes the established marketing strategy, since this module is responsible for the identification of LS to build the profiles of the clients or users, such as adaptive presentation, adaptive navigation and adaptation to the level of knowledge. Also, the CBR agent establishes, according to the profile and availability of the immersive devices, which experiences and e-learning objects will be dynamically presented to the clients or users of the system.

The proposed e-Marketing platform based on immersive technologies will use the technology of intelligent agent. The implementation of the intelligent agents will be done using the JADE platform, which provides a container for each host in which the agents are executed, is compatible with several languages and ontologies. The proposed architecture includes six classes of agents: Customer modelling agent, Centralized communications agent (BlackboardAgent), Monitoring agent, Marketing strategies generating agent, Activity Manager and CBR agent. This agent is oriented to two specific tasks: (i) to provide the agent that generates experiences and virtual objects with a selection of contents and a structure of interactions that corresponds to the background and profile of the customer; and (ii) to collaborate with the "Activity Manager", in the selection of experiences, activities and resources that are more appropriate for interacting with the e-Marketing system. The general architecture of the multiagent model proposed is shown in figure 2 . 


\subsection{End User Module}

The End User module refers to the devices available to implement the marketing strategy. These devices and their characteristics determine the degree of immersion that can be achieved. Devices such as HMD can allow a high degree of immersion. It is also important to take into consideration that the information record regarding interaction and immersion should be used according to the evaluation metrics of the marketing strategy to determine its effectiveness and to rethink the new actions to be taken.

It is in this context that the implementation of this architecture involves the use of techniques such as Journey Maps (Norton \& Pine, 2013), defined as a qualitative and human-centered design to track and visualize the discrete actions, thoughts and feelings of an agent's experience. A journey map quantitatively guides coding because it divides an agent's journey into individual steps and storytelling concepts. The Storydoing technique, consists of telling a story that is coherent with the behavior of the one who instrumentalizes it, so that this does not remain a simple narration. Immersive marketing can have storydoing as a source of development.

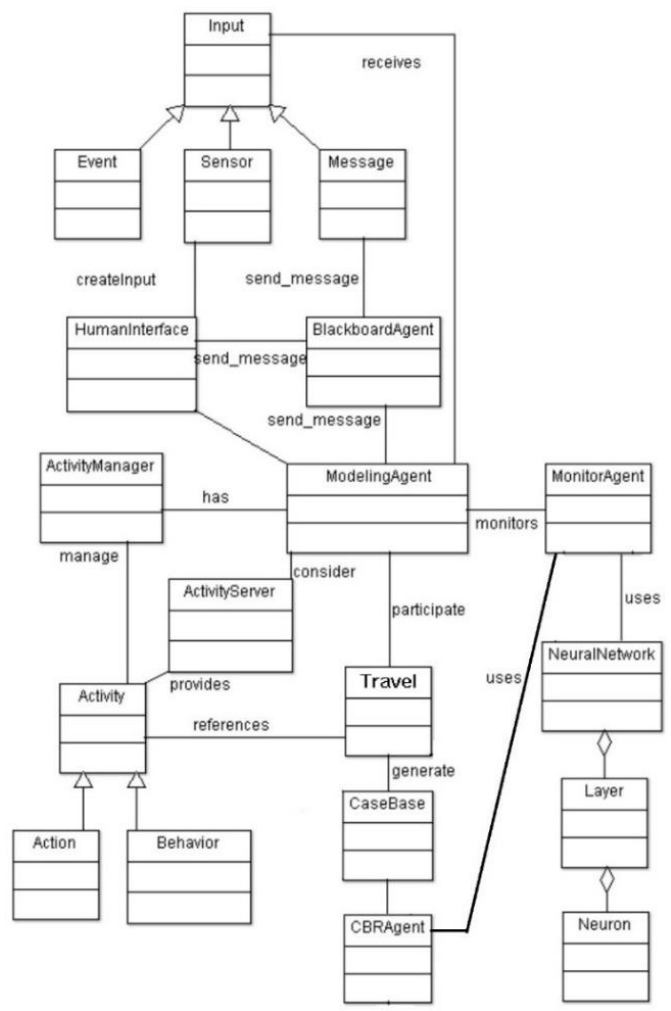

Figure 2. Multi-agent system architecture. Source: Alfaro, Rivera, \& Luna-Urquizo (2019)

\subsection{Materials and Methods}

Five phases were proposed for the development of this work: (i) User testing characterization, (ii) Theoretical framework, (iii) System Design, (iv) System Implementation, (v) Functional System evaluation. The model of Honey and Mumford (1992) was used for identifying learning styles; subsequently, a NeuroFuzzy Network was used for the online identification of LS, and the results were used for the assignment of learning experiences and objects through a CBR. In the system design phase, the concepts revised in theoretical framework were used in relation to Experiential Marketing, intelligent agents and VR, whose development process is described in the Software Architectural Proposal section. 
In the function of establishing the theoretical framework, the sampling and procedure for collecting bibliographic data was carried out through a search process that initially yielded 143 publications, which were summarized in a matrix of metadata for subsequent analysis. The quality assessment resulted in the retention of 66 publications. To ensure that the review process is rigorous and valid, the guidelines suggested by Webster and Watson (2002) and Okoli and Schabram (2010) have been followed.

\subsection{Experimental Design}

The neural network model tests were performed using an experimental method, having defined 20 resource categories (Table 1), among those available on the LMS Moodle, for the analysis and classification of users according to their LS and thus establish a record of their preferences derived from interactions with the system. Honey and Mumford (1992) surveys were applied to 34 undergraduate-level students using that LMS to obtain the required information, to correlate the LS obtained from the recording of interactions with the system, with those obtained from the surveys. Each resource categories were related to the four LS defined in the model, applying concepts of fuzzy logic, in relation to the characteristics associated with each LS.

Table 1. Resource categories and their relation to LS. Source: Alfaro, Rivera, Luna-Urquizo, Castañeda, \& Fialho (2018)

\begin{tabular}{|l|l|l|l|l|l|}
\hline & Resource type & Activist & Pragmatist & Reflector & Theorist \\
\hline 1 & Content (Text) & Low & Medium & High & High \\
\hline 2 & Content (Multimedia) & High & High & Medium & Medium \\
\hline 3 & Content (Mixed) & Medium & High & High & High \\
\hline 4 & Content (Simulation) & High & High & High & Medium \\
\hline 5 & Content (Urls) & Low & Low & High & High \\
\hline 6 & Examples (Text) & Medium & High & High & High \\
\hline 7 & Examples (Multimedia) & High & High & High & Medium \\
\hline 8 & Examples (Urls) & Medium & Low & High & High \\
\hline 9 & Case study (Text) & Medium & Medium & High & High \\
\hline 10 & Case study (Multimedia) & High & High & Medium & Medium \\
\hline 11 & Glossary (reading) & Low & Medium & High & High \\
\hline 12 & Glossary (writing) & Null & Low & High & High \\
\hline 13 & Wiki (reading) & Medium & Medium & High & Medium \\
\hline 14 & Wiki (writing) & Medium & Low & Medium & Low \\
\hline 15 & Chat (reading) & High & Medium & Low & Medium \\
\hline 16 & Chat (writing) & High & Medium & Low & Low \\
\hline 17 & Forum (reading) & Medium & Medium & Medium & Medium \\
\hline 18 & Forum (writing) & Medium & Medium & Low & Medium \\
\hline 19 & Self-assessments & Medium & Low & High & High \\
\hline 20 & Conceptual maps & Null & Medium & High & High \\
\hline
\end{tabular}

The results obtained by the neural network were satisfactory, compared with other approaches (Table 2).

Table 2. Comparison of evaluated models

\begin{tabular}{|l|c|c|}
\hline \multicolumn{1}{|c|}{ Evaluated models } & Learning Styles Model & Efficiency \\
\hline Bayesian Networks & Felder \& Silverman & $66 \%$ \\
\hline NB-Tree and CBR & Felder \& Silverman & $67.5 \%$ \\
\hline Monitoring of interactions & Felder \& Silverman & $79.6 \%$ \\
\hline Neural networks and navigation maps & Vincent \& Ross & $90 \%$ \\
\hline Learning objects and time estimation & Felder \& Silverman & $69.6 \%$ \\
\hline Stochastic models & Felder \& Silverman & $70 \%$ \\
\hline Neurofuzzy model (Proposal) & Honey \& Mumford & $77.1 \%$ \\
\hline
\end{tabular}

Likewise, CBR was used to assign learning experiences and objects to users according to marketing strategies, which can be established as a goal for a customer, with certain profiles and LS: What will be the most appropriate virtual experiences and objects for achieving the objectives of the marketing strategy?

The methodology used consists of five steps or tasks: Representation of a case, Case recovery, Reuse of cases, Case review and Case retention. For the tests of this module, 50 case retrieval queries were performed, 
which translates into tests of selection and assignment to clients and experiences, taking as the main search criteria the objective of the marketing strategy. These queries were carried out using a library of cases defined by specialists, obtaining that for $90 \%$ of the queries, the module could find at least 3 similar cases, and at least one of them with a similarity measure above $90 \%$.

\subsection{Hypothesis Testing}

The validation of hypotheses $\mathrm{H} 1$ and $\mathrm{H} 2$, was carried out through the review of experiential and sensory marketing literature, as well as AI techniques associated with immersive technologies, which allowed to conclude that if expectations are compared with the actual experiences of consumer perception, there is a differential that impacts on their memory and sensations, which influence the role that the mind unconsciously acquires. These aspects are incorporated in the design and conception of a "A Sensory Experiential Marketing System model applied to the Hotel Sector based on Immersive Technologies", which can contribute significantly to the attraction of potential customers in the hotel services sector.

To validate the H3 Hypothesis, a neuro-fuzzy network was developed to identify users' LS, which processes information from user interactions with the system online, the results of which were correlated with those obtained from the application of the LS classification survey. The results obtained by the neural network were satisfactory, compared to the resolution performed with other approaches as shown in Table 2 .

The validation of $\mathrm{H} 4$, also carried out through bibliographic research, in relation to the contribution of the effectiveness of immersive technologies in hotel marketing activities and strategies, allowed to establish that this type of technological solution for tourism applications can be fully immersive, semi-immersive and non-immersive, It is a good solution for tourism marketing activities using VR, highlighting that Natural User Interfaces (NUI) allow interaction with the user in a natural, common and familiar way, which implies the use of new interaction devices that are intuitive and easy to use, unlike traditional interfaces.

To validate CBR system, 50 case recovery queries were performed, resulting in customer selection and assignment tests, and experiences. These queries were conducted using a specialist-defined case library, obtaining that for $90 \%$ of the queries, the module could find at least 3 similar cases, and at least one of them with a similarity measure greater than $90 \%$, allowing to establish that satisfactory results were obtained.

\section{CONCLUSION}

The state of the art of Experience Marketing of the adaptive systems was established, as well as emerging immersive technologies and their applications in Marketing for the proposal of a theoretical model of adaptive e-Marketing, allowing to focus on the enormous potential of these technologies to generate experiences that include multisensory perceptions, which can awaken unique emotions and sensations in visitors or users of websites. This study can provide a theoretical basis, to allow to offer pleasant virtual experiences and objects, using immersive technologies, in order to increase the level of customer satisfaction focused on the perception of the quality of service of the hotel's websites.

A hybrid adaptive e-Learning software architecture has been proposed, incorporating various AI approaches and techniques, oriented to hotel services marketing, based on immersive technologies whose attributes of originality and innovation are unique, due to the incorporation of LS to achieve an adaptive approach, according to guest or user profiles. The implementation in practical cases of hotel marketing will involve the use of Journey Maps, to track and visualize the actions, thoughts and discrete feelings of the experience of an agent, as well as the use of techniques such as storytelling and storydoing that will allow to tell stories that do not remain in a simple narrative, allowing a certain degree of participation and interaction of the future guest with the system, which will improve the quality of interaction, allowing the "visit" prior to the reservation or sale, of the facilities, green areas, services and the very environment of the hotel company.

The results of this study should be carefully interpreted for the future development of the architectural proposal. Some limitations, such as data unavailability and inaccessibility, researcher time and budget constraints, and some other practical concerns, have not yet been established. Recognizing these limitations, we suggest other possible determinants to observe, such as booking intent, room rate strategy, etc., which can be important factors influencing the performance of the proposed model and customer satisfaction. 


\section{REFERENCES}

Al-Chalabi, H., \& Hussein, A. (2020). Ontologies and Personalization Parameters in Adaptive E-learning Systems: Review. Journal of Applied Computer Science \& Mathematics, 14(1), 14-19.

Alagöz, S. B., \& Ekici, N. (2014). Experiential Marketing and Vacation Experience: The Sample of Turkish Airlines*. Procedia - Social and Behavioral Sciences, 150, 500-510. https://doi.org/10.1016/j.sbspro.2014.09.065

Alfaro, L., Rivera, C., \& Luna-Urquizo, J. (2019). Using Project-based learning in a Hybrid e-Learning system model. International Journal of Advanced Computer Science and Applications, 10(10), 426-436. https://doi.org/10.14569/ijacsa.2019.0101059

Alfaro, L., Rivera, C., Luna-Urquizo, J., Alfaro, S., \& Fialho, F. (2019). Knowledge construction by immersion in virtual reality environments. International Journal of Advanced Computer Science and Applications, 10(12). https://doi.org/10.14569/ijacsa.2019.0101278

Alfaro, L., Rivera, C., Luna-Urquizo, J., Castañeda, E., \& Fialho, F. (2018). Fuzzy neural system model for online learning styles identification, as an adaptive hybrid e-learning system architecture component. Proceedings of the LACCEI International Multi-Conference for Engineering, Education and Technology, 2018-July, 1-6. https://doi.org/10.18687/laccei2018.1.1.259

Alfaro, L., Rivera, C., Luna-Urquizo, J., Zuńiga, J. C., Portocarrero, A., \& Raposo, A. B. (2019). Immersive technologies in marketing: State of the Art and a Software Architecture Proposal. International Journal of Advanced Computer Science and Applications, 10(10), 482-490. https://doi.org/10.14569/ijacsa.2019.0101064

Alfaro, Luis, Rivera, C., Castãneda, E., Zũniga-Cueva, J., Rivera-Chavez, M., \& Fialho, F. (2020). A review of intelligent tutorial systems in computer and web based education. International Journal of Advanced Computer Science and Applications, 11(2), 755-763. https://doi.org/10.14569/ijacsa.2020.0110295

Beck, J., Rainoldi, M., \& Egger, R. (2019). Virtual reality in tourism: a state-of-the-art review. Tourism Review, 74(3), 586-612. https://doi.org/10.1108/TR-03-2017-0049

Botsios, S., \& Georgiou, D. (2008). Recent adaptive e-learning contributions towards a "standard ready" architecture. MCCSIS'08 - IADIS Multi Conference on Computer Science and Information Systems; Proceedings of e-Learning 2008, 2, 226-230. Amsterdam, The Netherlands.

Burdea, G., \& Coiffet, P. (2003). Virtual Reality Technology. John Wiley \& Sons.

Carbonell, J., Sánchez-Esguevillas, A., \& Carro, B. (2016). The role of metaphors in the development of technologies. The case of the artificial intelligence. Futures, 84, 145-153. https://doi.org/10.1016/j.futures.2016.03.019

Esichaikul, V., Lamnoi, S., \& Bechter, C. (2011). Student modelling in adaptive e-learning systems. Knowledge Management and E-Learning, 3(3), 342-355. https://doi.org/10.34105/j.kmel.2011.03.025

Gallo, I., Townsend, C., \& Alegre, I. (2019). Experiential product framing and its influence on the creation of consumer reviews. Journal of Business Research, 98, 177-190. https://doi.org/10.1016/j.jbusres.2019.01.007

Grubišić, A., Stankov, S., \& Žitko, B. (2015). Adaptive courseware: A literature review. Journal of Universal Computer Science, 21(9), 1168-1209. https://doi.org/10.3217/jucs-021-09-1168

Gutiérrez A., M. A., Vexo, F., \& Thalmann, D. (2008). Stepping into virtual reality. In Stepping into Virtual Reality. London: Springer-Verlag. https://doi.org/10.1007/978-1-84800-117-6

Hoyer, W. D., Kroschke, M., Schmitt, B., Kraume, K., \& Shankar, V. (2020). Transforming the Customer Experience Through New Technologies. Journal of Interactive Marketing, 51, 57-71.

Hwangbo, H., Kim, Y. S., \& Cha, K. J. (2017). Use of the Smart Store for Persuasive Marketing and Immersive Customer Experiences: A Case Study of Korean Apparel Enterprise. Mobile Information Systems, 2017, 1-17.

Krishna, A. (2012). An integrative review of sensory marketing: Engaging the senses to affect perception, judgment and behavior. Journal of Consumer Psychology, 22(3), 332-351. https://doi.org/10.1016/j.jcps.2011.08.003

Kumar, V., Ramachandran, D., \& Kumar, B. (2020). Influence of new-age technologies on marketing: A research agenda. Journal of Business Research. https://doi.org/10.1016/j.jbusres.2020.01.007

Lewis Johnson, W., \& Lester, J. C. (2018). Pedagogical agents: Back to the future. AI Magazine, 39(2), 33-44.

Norton, D. W., \& Pine, B. J. (2013). Using the customer journey to road test and refine the business model. Strategy and Leadership, 41(2), 12-17. https://doi.org/10.1108/10878571311318196

Parasuraman, A., Zeithaml, V. A., \& Berry, L. L. (2018). A Conceptual Model of Service Quality and Its Implications for Future Research. Journal of Marketing, 49(4), 41-50. https://doi.org/10.1177/002224298504900403

Shute, V., \& Towle, B. (2003). Adaptive e-learning. Educational Psychologist, 38(2), $105-114$. https://doi.org/10.1207/S15326985EP3802_5

Soni, N., Sharma, E. K., Singh, N., \& Kapoor, A. (2020). Artificial Intelligence in Business: From Research and Innovation to Market Deployment. Procedia Computer Science, 167, 2200-2210.

Triantafillou, E., Pomportsis, A., \& Georgiadou, E. (2002). AES-CS: adaptive educational system based on cognitive styles. Proceedings of AH2002 Workshop, Second International Conference on Adaptive Hypermedia and Adaptive Web-Based Systems, 10-21. Malaga, Spain.

Verhoef, P. C., Lemon, K. N., Parasuraman, A., Roggeveen, A., Tsiros, M., \& Schlesinger, L. A. (2009). Customer Experience Creation: Determinants, Dynamics and Management Strategies. Journal of Retailing, 85(1), 31-41.

Winn, W. (1993). A Conceptual Basis for Educational Applications of Virtual Reality. In Washington Technology Centre University of. Seatle: University of Washington. 